\title{
Caracterização morfoestrutural de fêmeas e machos jovens de ovinos naturalizados Sul-mato-grossenses "Pantaneiros"
}

\section{Morphostructural characterization of female and young male of naturalized Sul-mato-grossenses "Pantaneiros" sheep}

\author{
Daniele Portela de Oliveira ${ }^{1 *}$; Carlos Antonio Lopes de Oliveira ${ }^{2}$; \\ Elias Nunes Martins'; Fernando Miranda Vargas Junior; \\ Marcos Barbosa-Ferreira'; Leonardo Oliveira Seno ${ }^{3}$; \\ Junior Cezar Kawakita de Oliveira ${ }^{5}$ Aya Sasa ${ }^{6}$
}

\begin{abstract}
Resumo
Medidas morfométricas são importantes para avaliar a variabilidade, determinar o padrão racial e a existência de dimorfismo sexual em um grupo genético. Com este objetivo foram realizadas 20 medidas morfométricas e uma medida ponderal em 338 animais do grupo genético de ovinos naturalizados Sulmato-grossenses pertencentes à Fundação Manoel de Barros, criadas no Centro Tecnológico de Ovinos (CTO) da Universidade Anhanguera-Uniderp localizado em Campo Grande-MS e a Universidade Federal da Grande Dourados. As análises estatísticas foram realizadas por meio do software SAS. As ovelhas e os machos jovens apresentaram baixa variabilidade para as medidas morfométricas da região cefálica e membros. Porém, maior variabilidade foi verificada nas medidas morfométricas tomadas da região do tronco indicando que esta região poderia ser utilizada como critério de seleção em programas de melhoramento. Os coeficientes de correlação de Pearson entre as características morfoestruturais, tanto para ovelhas quanto para machos jovens, apontam um rebanho com certo grau de harmonia no modelo morfoestrutural. As fêmeas caracterizam-se por animais com cabeça relativamente grande, com características do tronco proporcionais e bons aprumos. O gênero não influenciou as características da região cefálica e o peso corporal nos animais jovens. No entanto, as características das outras regiões, o perímetro do pescoço e a largura posterior de garupa mensuradas na região do tronco, e o perímetro do carpo e metacarpo nos membros foram influenciados pelo gênero no animal jovem.
\end{abstract}

Palavras-chave: Correlação, morfometria, ovinos, raças naturalizadas

\footnotetext{
${ }^{1}$ Discente do Programa de Pós-Graduação em Zootecnia, Universidade Estadual de Maringá, UEM, Maringá, PR. E-mail: dane_ portela@yahoo.com.br

2 Prof. do Dept ${ }^{\circ}$ de Zootecnia, UEM, Maringá, PR. E-mail: caloliveira@uem.br; enmartins@uem.br

${ }^{3}$ Profs. do Programa de Pós-Graduação em Zootecnia, Universidade Federal da Grande Dourados, UFGD, Dourados, MS. E-mail: fernandojunior@ufgd.edu.br; leonardoseno@ufgd.edu.br

${ }^{4}$ Prof. do Curso de Mestrado em Produção e Gestão Agroindustrial, Universidade Anhanguera, UNIDERP, Campo Grande, MS. E-mail: profmarcosvet@gmail.com

${ }^{5}$ Discente do curso de Medicina Veterinária, Faculdade de Medicina Veterinária e Zootecnia, UFMS, Campo Grande, MS. Bolsista Embrapa Gado de Corte. E-mail: juniorkawakita@hotmail.com

${ }^{6}$ Coordenadora do Programa de Pós-Graduação em Zootecnia, Universidade Estadual de Mato Grosso do Sul, UEMS, Aquidauana, MS.E-mail: aya@uems.br

* Autor para correspondência
} 


\begin{abstract}
Morphometric measurements are important to evaluate the variability, determine the racial standard and the existence of sexual dimorphism in genetic group. With this objective, were measured 20 morphometric measurements and one measurements of weight for 338 animals of the genetic group of Sul-mato-grossenses naturalized sheep belonging to Manoel de Barros Foundation and breeding at Centro Tecnologico de Ovinos from Anhanguera-Uniderp University and Federal University of the Grande Dourados. The statistical analyses were realized through software SAS. The females and young males showed short variation for morphometric measured of head and limbs region. However, higher variation was observed in the morphometric measurements taken from torso region, indicate that this region could be inserted in breeding programs like a selection criterion. The Pearson correlation coefficients between the morphostructural characteristics of females and young males, appears to have certain harmony degree in the morphostructural model. The females are characterized by animals with relatively large head, torso with proportional features and good angulations. The gender did not influence characteristics of the head region and body weight in the young animals. However, the characteristics of other regions, such as thoracic perimeter and posterior width of rump measured in the head region, and the perimeter of carpal and metacarpal in the limbs were influenced by gender in young animals.

Key words: Correlation, morphometric, naturalized breeds, sheep
\end{abstract}

\section{Introdução}

No Mato Grosso do Sul são relatadas informações acerca de um grupo genético de ovinos, denominados "pantaneiros" ou nativos Sulmato-grossenses, adaptados às condições climáticas da região. Os ovinos pantaneiros são oriundos de cruzamentos entre as raças que foram trazidas pelos colonizadores portugueses e espanhóis logo após o descobrimento (MARIANTE et al., 1999).

No ano de 2005, foi iniciado um estudo exploratório por pesquisadores da Uniderp - CTO, Embrapa, UFMS e, posteriormente, a UFGD, a fim de identificar e manter o grupamento genético, para evitar a extinção, pelo risco desta raça ser substituída por raças exóticas (VARGAS JUNIOR et al., 2011a). O grupamento genético de ovinos naturalizados Sul-mato-grossenses apresenta uma combinação de alelos que se aproximam de raças lanadas do Sul e deslanadas do Nordeste (GOMES et al., 2007). Estes animais são encontrados em fazendas mais isoladas da região, sem nenhum controle reprodutivo ou sanitário, vivendo há muitos anos praticamente sob seleção natural, fato este que possibilita concluir que esses ovinos são adaptados à região Sul-mato-grossense (VARGAS JUNIOR et al., 2011b).

Para o conhecimento das características biométricas e funcionais das raças naturalizadas são necessários estudos que possibilitem a real avaliação da variabilidade genética existente entre e dentro das espécies, mostrando suas possibilidades de uso nos programas de melhoramento (McMANUS, 2005). O estudo da morfologia, amplamente coberta nos padrões raciais, requer extrema objetividade, o que é alcançado pela caracterização biométrica da estrutura óssea dos animais (BLANCO et al., 1990).

Essa caracterização pode ser realizada por meio de medidas morfométricas, coloração dos animais, índices zootécnicos e desempenho de acordo com o gênero e categoria. Além disso, contribui cada vez mais para o conhecimento da conformação dos indivíduos que constituem cada grupamento genético e para o estabelecimento da relação entre conformação e função do animal (SILVA et al., 2007).

Assim, o objetivo da realização desse trabalho foi a caracterização morfoestrutural do grupo genético de ovinos naturalizados Sul-mato-grossenses por meio de medidas morfométricas quantitativas e uma medida ponderal. 


\section{Material e Métodos}

A caracterização morfoestrutural realizou-se com base em medidas morfométricas de animais de ambos os gêneros, sendo 296 fêmeas e 42 machos jovens, pertencentes à Fundação Manoel de Barros, criadas no Centro Tecnológico de Ovinos (CTO) da Universidade Anhanguera-Uniderp localizado em Campo Grande-MS e a Universidade Federal da Grande Dourados-MS. As fêmeas amostradas apresentaram cronologia dentária que variou de dente de leite a oito dentes permanentes, e os cordeiros jovens apresentaram somente dente de leite, ou seja, antes da primeira muda de dentes.

Um total de 20 medidas lineares morfoestruturais e uma medida ponderal foram tomadas de acordo com a metodologia adotada por vários autores, como Blanco et al. (1990), Herrera et al. (1996),
Casanova; Vidal (2007) e Torres (2002).

As variáveis morfoestruturais foram agrupadas de acordo com as regiões do corpo dos animais, divididas em medidas cefálicas, no tronco e dos membros do corpo, conforme descrito abaixo:

- Medidas cefálicas

1. Longitude ou comprimento da cabeça (LCB): medida entre a extremidade caudal do occipital e o ponto mais rostral do crânio (osso incisivo). 2. Comprimento do crânio (CCr): distância vertical entre a nuca e a parte mediana ou central da arcada incisiva inferior. 3. Longitude do rosto (LR): medida entre a linha imaginária que une o ângulo interno dos olhos e o ponto mais rostral da região nasal. 4. Largura da cabeça (LC): medida entre os arcos zigomáticos. 5. Tamanho da orelha (TO): medida da base da orelha até a extremidade da pina (Figura 1).

Figura 1. Ilustrações da vista de perfil (a) e de frente (b) das medidas realizadas na região cefálica.

a)

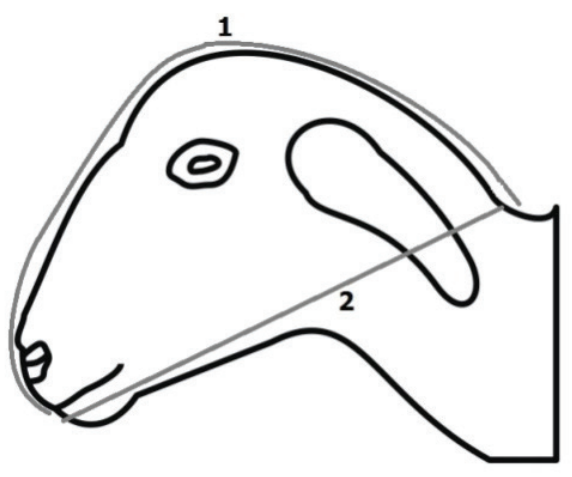

Fonte: Elaboração dos autores.

- Medidas no tronco

6. Perímetro do pescoço (PP): medida do perímetro do pescoço. 7. Comprimento do pescoço (CP): distância entre o centro da nuca no occipital e o meio da cernelha. 8. Comprimento do corpo (CC): medida da articulação escápula-umeral até a extremidade caudal do ísqueo. 9. Diâmetro dorsoesternal (DE): distância vertical entre a região craniocaudal do dorso e o esterno. 10. Largura b)

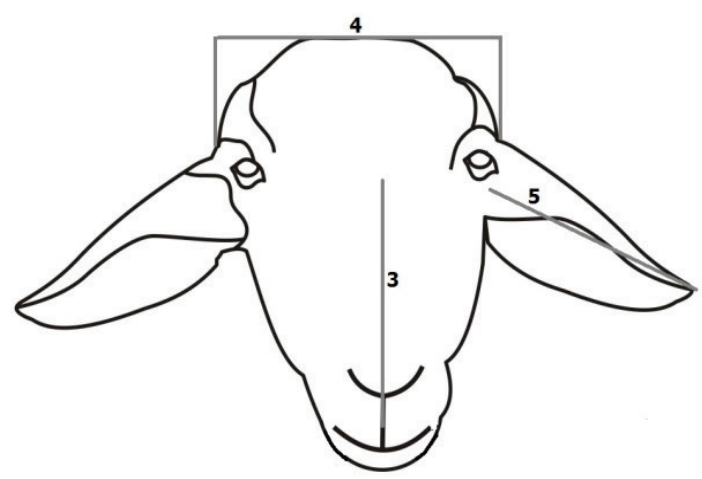

entre ombros (LO): medida compreendida entre a ponta das escápulas. 11. Perímetro torácico $(\mathrm{PT})$ : medida que parte do ponto de declividade da região interescapular, envolvendo toda região torácica. 12. Largura da garupa (LG): distância entre a região cranial da tuberosidade ilíaca e a caudal da tuberosidade isquiática. 13. Largura anterior da garupa $(\mathrm{LaG})$ : distância entre as regiões craniais das tuberosidades ilíacas. 14. Largura posterior da 
garupa (LpG): distância entre as regiões caudais das tuberosidades isquiáticas. 15. Comprimento de tetos (CT): medida desde a base do teto no úbere até a extremidade distal (Figura 2).

Figura 2. Ilustração das medidas na região do tronco e membros no lado esquerdo do animal.

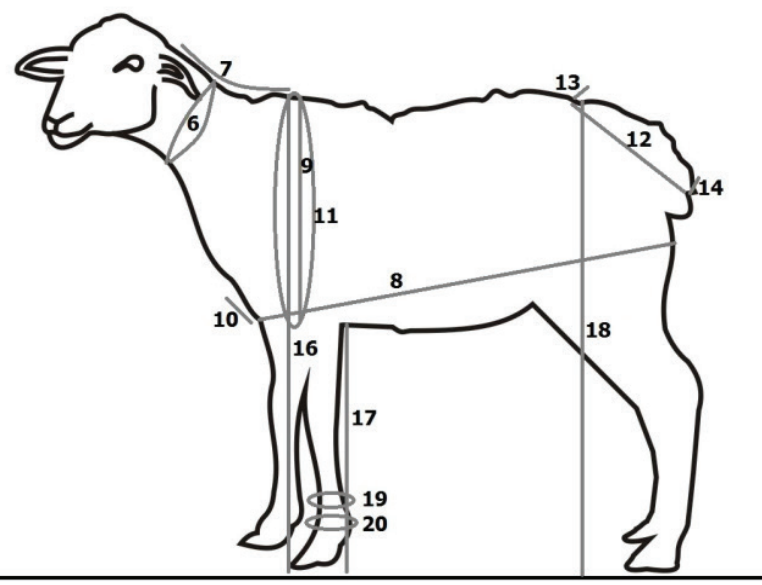

Fonte: Elaboração dos autores.

- Medidas dos membros

16. Altura da cernelha (AC): distância vertical do ponto mais alto da região interescapular até o solo. 17. Altura da região subesternal (ARE): distância vertical da região abaixo do esterno ao apoio do animal. 18. Altura de garupa (AG): distância vertical do ponto mais alto da garupa, no espaço definido pelo processo espinhoso de T5 - T6 sobre a tuberosidade sacral do íleo, até o solo. 19. Perímetro do carpo (PC): medida da articulação do carpo. 20. Perímetro do metacarpo (PM): medida do terço médio da região metacarpiana do membro torácico esquerdo (Figura 2).

- Medida ponderal

\section{Peso corporal.}

Foram obtidas as medidas $1,3,6,7,11,19$ e 20 com fita métrica; as medidas 2,4,5,12,13,14 e 15 com paquímetro; e as medidas 8,9,10,16,17 e 18 com bastão zoométrico. O peso corporal de todos os animais foi obtido com balança móvel para ovinos. As medidas foram tomadas sempre do lado esquerdo do animal.

Foi realizada análise descritiva geral e a estimativa de correlações de Pearson de ovelhas de todas as classes de idade e somente machos jovens com a ocorrência antes da primeira muda de dentes, analisados separadamente de acordo com o gênero. $\mathrm{Na}$ análise descritiva geral das características mensuradas foram calculados a média, o mínimo, o máximo, o desvio-padrão e o coeficiente de variação e as correlações de Pearson, por meio do procedimento MEAN e CORR do software Statistical Analyses System (SAS, 2002).

Para avaliar as diferenças estatísticas para as características morfoestruturais, no crescimento das ovelhas, elas foram agrupadas de acordo com a classe de idade (Costa Júnior et al., 2006). A análise estatística dos dados foi realizada utilizando-se o Método de Quadrados Mínimos e as diferenças entre médias pelo teste Tukey a $(\mathrm{P}<0,05)$, por meio do procedimento GLM do software Statistical Analyses System (SAS, 2002).

Para avaliar o dimorfismo sexual entre cordeiros para as características morfoestruturais mensuradas, foram utilizados somente animais, machos e fêmeas, da primeira classe de idade (dente de leite). A análise estatística dos dados foi realizada utilizando-se o Método de Quadrados Mínimos, por meio do procedimento GLM do software Statistical Analyses System (SAS, 2002). No modelo estatístico foi considerado somente o efeito de gênero e o erro aleatório de cada variável mensurada.

\section{Resultados e Discussão}

Analise descritiva das características morfoestruturais

- Medidas cefálicas

As características mensuradas na região cefálica 
tanto para fêmeas quanto para machos jovens apresentaram coeficientes de variação em torno de $10 \%$, o que é considerado razoável a bom (OLIVEIRA et al., 2007) para o grupo genético (Tabela 1). No grupo de fêmeas, os coeficientes de variação mais altos são observados pelas diferenças na idade cronológica (GINÉS, 2009).

Segundo Abud et al. (2011), características como comprimento da cabeça e largura da cabeça estão ligadas ao desenvolvimento dos animais e a caracterização racial. Consistem em medidas que demonstram o crescimento do animal e tem menor influência do meio podendo ser utilizadas para a padronização racial, contudo pequena importância para a produção animal.

- Medidas no tronco

Foi observado elevado coeficiente de variação tanto para fêmeas como para os machos jovens em algumas características mensuradas no tronco (Tabela 1). De acordo com Torres (2002), há vários fatores que podem condicionar a tomada das medidas dos animais, que por sua vez, podem influenciar na precisão das mesmas e, consequentemente, nos resultados como a condição corporal e os diferentes estágios de desenvolvimento.

A condição corporal e os diferentes estágios de desenvolvimento corporal dos animais podem ter sido responsáveis pelos elevados coeficientes de variação para largura anterior e posterior de garupa e comprimento de tetos nas fêmeas, por serem regiões anatômicas passíveis de mudanças com a condição fisiológica do animal. Todavia, Cuesta (2009) afirma que a observação de elevados coeficientes de variação em algumas medidas morfométricas indica que são regiões com muita variabilidade, portanto, objeto de seleção para diminuir a variação e dar maior uniformidade à população, caso seja de interesse, ou sirva para explicar cruzamentos, adaptações a diferentes sistemas de cria dentro da população.

- Medidas dos membros

As características mensuradas na região dos membros (Tabela 1) para fêmeas e machos jovens apresentaram coeficientes de variação abaixo de $10 \%$ exceto para perímetro do metacarpo nos cordeiros $(\mathrm{CV}=11,8 \%)$. De acordo com Oliveira et al. (2007), coeficientes de variação próximos de 10\% são considerados razoáveis a bons do ponto de vista estatístico, indicando que o grupo genético apresenta certa harmonia para tais características. Blanco et al. (1990) observaram variação semelhante para as medidas nos membros em ovinos da raça Segureña.

- Medida ponderal

No peso corporal (Tabela 1), tanto de fêmeas quanto nos machos jovens, foi observado elevado coeficiente de variação. O peso corporal foi a característica que apresentou maior variação para os dois gêneros, mostrando ser mais influenciado pelo ambiente, conforme constatado por Costa Júnior et al. (2006). Blanco et al. (1990) observaram coeficiente de variação de $20,5 \%$ para machos e $1,5 \%$ para fêmeas. 
Tabela 1. Analise descritiva média, mínimo, máximo, desvio padrão (DP) e coeficiente de variação (CV) das variáveis morfoestruturais, em centímetros, para fêmeas (F) e machos jovens (M) dos ovinos naturalizados Sul-mato-grossenses.

\begin{tabular}{|c|c|c|c|c|c|c|}
\hline Variáveis & Gênero & Média & Mínimo & Máximo & $\mathrm{DP}$ & $\mathrm{CV}(\%)$ \\
\hline \multirow[t]{2}{*}{ Longitude da cabeça } & $\mathrm{F}$ & 32,4 & 21,8 & 37,0 & 2,4 & 7,5 \\
\hline & M & 31,2 & 24,0 & 39,5 & 3,4 & 10,8 \\
\hline \multirow[t]{2}{*}{ Comprimento do crânio } & $\mathrm{F}$ & 24,2 & 15,2 & 28,2 & 2,3 & 9,7 \\
\hline & M & 21,7 & 17,8 & 25,4 & 1,8 & 8,5 \\
\hline \multirow[t]{2}{*}{ Longitude do rosto } & $\mathrm{F}$ & 13,7 & 8,0 & 17,5 & 1,5 & 10,7 \\
\hline & M & 12,0 & 8,5 & 15,0 & 1,5 & 12,4 \\
\hline \multirow[t]{2}{*}{ Largura da cabeça } & $\mathrm{F}$ & 9,4 & 7,0 & 11,9 & 0,9 & 9,3 \\
\hline & M & 8,8 & 7,7 & 10,4 & 0,6 & 7,36 \\
\hline \multirow[t]{2}{*}{ Tamanho da orelha } & $\mathrm{F}$ & 14,3 & 8,9 & 18,5 & 1,5 & 10,7 \\
\hline & M & 14,1 & 11,8 & 16,8 & 1,3 & 9,1 \\
\hline \multirow[t]{2}{*}{ Perímetro do pescoço } & $\mathrm{F}$ & 28,5 & 17,0 & 40,5 & 3,3 & 11,5 \\
\hline & M & 30,1 & 23,0 & 38,0 & 4,1 & 13,8 \\
\hline \multirow[t]{2}{*}{ Comprimento do pescoço } & $\mathrm{F}$ & 25,7 & 12,0 & 33,0 & 2,7 & 10,7 \\
\hline & M & 24,2 & 19,5 & 28,0 & 1,8 & 7,5 \\
\hline \multirow[t]{2}{*}{ Comprimento do corpo } & $\mathrm{F}$ & 63,6 & 41,5 & 75,5 & 5,8 & 9,2 \\
\hline & M & 59,9 & 48,6 & 76,0 & 6,3 & 10,5 \\
\hline \multirow[t]{2}{*}{ Diâmetro dorso-esternal } & $\mathrm{F}$ & 27,3 & 18,0 & 33,5 & 2,8 & 10,3 \\
\hline & M & 24,9 & 19,0 & 31,0 & 2,4 & 9,9 \\
\hline \multirow[t]{2}{*}{ Largura entre ombros } & $\mathrm{F}$ & 16,4 & 10,3 & 21,2 & 1,8 & 10,8 \\
\hline & M & 16,2 & 12,1 & 20,3 & 2,0 & 12,6 \\
\hline \multirow[t]{2}{*}{ Perímetro torácico } & $\mathrm{F}$ & 76,0 & 37,0 & 96,0 & 7,5 & 9,9 \\
\hline & M & 71,2 & 55,5 & 98,5 & 8,9 & 12,5 \\
\hline \multirow[t]{2}{*}{ Largura da garupa } & $\mathrm{F}$ & 20,6 & 12,9 & 24,4 & 2,0 & 10,0 \\
\hline & M & 18,9 & 14,9 & 23,6 & 2,0 & 10,5 \\
\hline \multirow[t]{2}{*}{ Largura anterior da garupa } & $\mathrm{F}$ & 15,5 & 9,0 & 21,1 & 2,4 & 15,4 \\
\hline & M & 13,2 & 10,3 & 16,4 & 1,6 & 12,6 \\
\hline \multirow[t]{2}{*}{ Largura posterior da garupa } & $\mathrm{F}$ & 5,4 & 2,5 & 7,7 & 0,9 & 17,0 \\
\hline & M & 4,3 & 3,1 & 6,2 & 0,7 & 17,2 \\
\hline \multirow[t]{2}{*}{ Comprimento de tetos } & $\mathrm{F}$ & 1,7 & 0,4 & 3,3 & 0,4 & 24,8 \\
\hline & M & - & - & - & - & - \\
\hline \multirow[t]{2}{*}{ Altura da cernelha } & $\mathrm{F}$ & 63,6 & 45,2 & 84,2 & 4,8 & 7,5 \\
\hline & M & 60,2 & 50,7 & 71,7 & 4,9 & 8,0 \\
\hline \multirow[t]{2}{*}{ Altura de garupa } & $\mathrm{F}$ & 65,5 & 47,2 & 86,7 & 5,2 & 7,8 \\
\hline & M & 61,3 & 52,7 & 72,7 & 4,8 & 7,8 \\
\hline \multirow[t]{2}{*}{ Altura da região subesternal } & $\mathrm{F}$ & 36,4 & 27,2 & 52,7 & 3,0 & 8,2 \\
\hline & M & 35,1 & 28,7 & 41,2 & 3,4 & 9,8 \\
\hline \multirow[t]{2}{*}{ Perímetro do carpo } & $\mathrm{F}$ & 12,2 & 9,5 & 14,0 & 0,7 & 6,2 \\
\hline & M & 12,6 & 10,5 & 14,5 & 1,2 & 9,7 \\
\hline \multirow[t]{2}{*}{ Perímetro do metacarpo } & $\mathrm{F}$ & 7,2 & 5,2 & 8,5 & 0,5 & 7,5 \\
\hline & M & 7,5 & 6,0 & 9,0 & 0,9 & 11,8 \\
\hline \multirow[t]{2}{*}{ Peso corporal } & $\mathrm{F}$ & 32,4 & 10,5 & 56,0 & 7,9 & 24,3 \\
\hline & M & 29,5 & 14,0 & 51,5 & 9,0 & 30,6 \\
\hline
\end{tabular}

Fonte: Elaboração dos autores. 
Coeficientes de correlação entre as características morfoestruturais

Todos os coeficientes de correlação (Tabela 2) resultaram em valores positivos, tanto para fêmeas quanto para machos jovens. As variáveis morfoestruturais da região cefálica apresentaram moderada a forte associação entre elas, tanto em fêmeas como nos machos jovens, exceto para tamanho de orelha (TO) nas fêmeas.

De modo geral, a região do tronco apresentou moderada associação entre elas, tanto em fêmeas como em machos jovens. Nas fêmeas, destaca-se a pouca associação do perímetro do pescoço (PP) com a região do tronco, exceto com largura entre ombros (LO), onde apresentou uma moderada associação $(0,53)$. Já para os machos jovens, observou-se forte associação entre largura de garupa (LG) e diâmetro dorso-esternal (DE). Ao contrário delas, o comprimento do pescoço (CP) foi pouco associado com PP, perímetro torácico $(\mathrm{PT})$, largura anterior de garupa $(\mathrm{LaG})$, largura posterior de garupa (LpG) e também entre PT e LpG.

Na região do tronco, nas fêmeas, observou-se que o comprimento do corpo (CC) com DE, PT, largura de garupa (LG) e LaG; DE com PT, LG, LaG; PT com $\mathrm{LG}$ e $\mathrm{LaG}$, e $\mathrm{LaG}$ com $\mathrm{LG}$ possuem forte associação que variam de 0,74 a 0,86 . Indicando que as fêmeas têm desenvolvimento corporal harmonioso com perfil para produção de carne. Oliveira et al. (2007) observaram associação fraca e negativa para PT com LG, sugerindo que as ovelhas Texel criadas na parte alta do Pantanal necessitam reverter tal situação, pois estas características estão associadas à produção de cortes nobres de carne.
Na região dos membros das fêmeas, observouse associação muito variável $(0,18-0,95)$ entre as variáveis mensuradas. Cabendo destacar que a altura de cernelha (AC) possuiu uma associação muito forte com a altura de garupa (AG), no entanto, foi fracamente associada com perímetro do metacarpo (PM). A altura da região subesternal (ARE) possui fraca associação com PC e PM, porém, foi fortemente associada com AC e AG. De acordo com Abud et al. (2011), as características AC e AG são de interesse econômico, por estarem ligadas à produtividade animal.

Para os machos jovens, as variáveis mensuradas apresentaram moderada a forte associação entre elas. Foi observado entre $\mathrm{AC}$ e $\mathrm{AG}$, correlação de 0.95. Carneiro (2008) observou valores de correlação entre AC e AG de 0,42 para raças ovinas no Brasil, Uruguai e Colômbia. Todavia, Torres (2002) observou em machos da raça Xisqueta valor de 0,75 para associação entre $\mathrm{AC}$ e AG.

O peso corporal, medida ponderal observada nas fêmeas, possui associação que varia de fraca a forte magnitude $(0,20-0,85)$. Na região cefálica, o tamanho de orelha (TO) é fracamente associado com o peso corporal, diferentemente das outras variáveis. O TO tem fraca associação com todas as variáveis morfoestruturais e com o peso corporal, indicando que esta característica sofrerá pouca influência com a seleção dos animais para alguma das características morfoestruturais mensuradas ou para peso corporal. Na região dos membros, observa-se moderada associação entre as variáveis e o peso corporal, exceto para a fraca associação observada entre peso corporal e ARE com valor de 0,24 . 


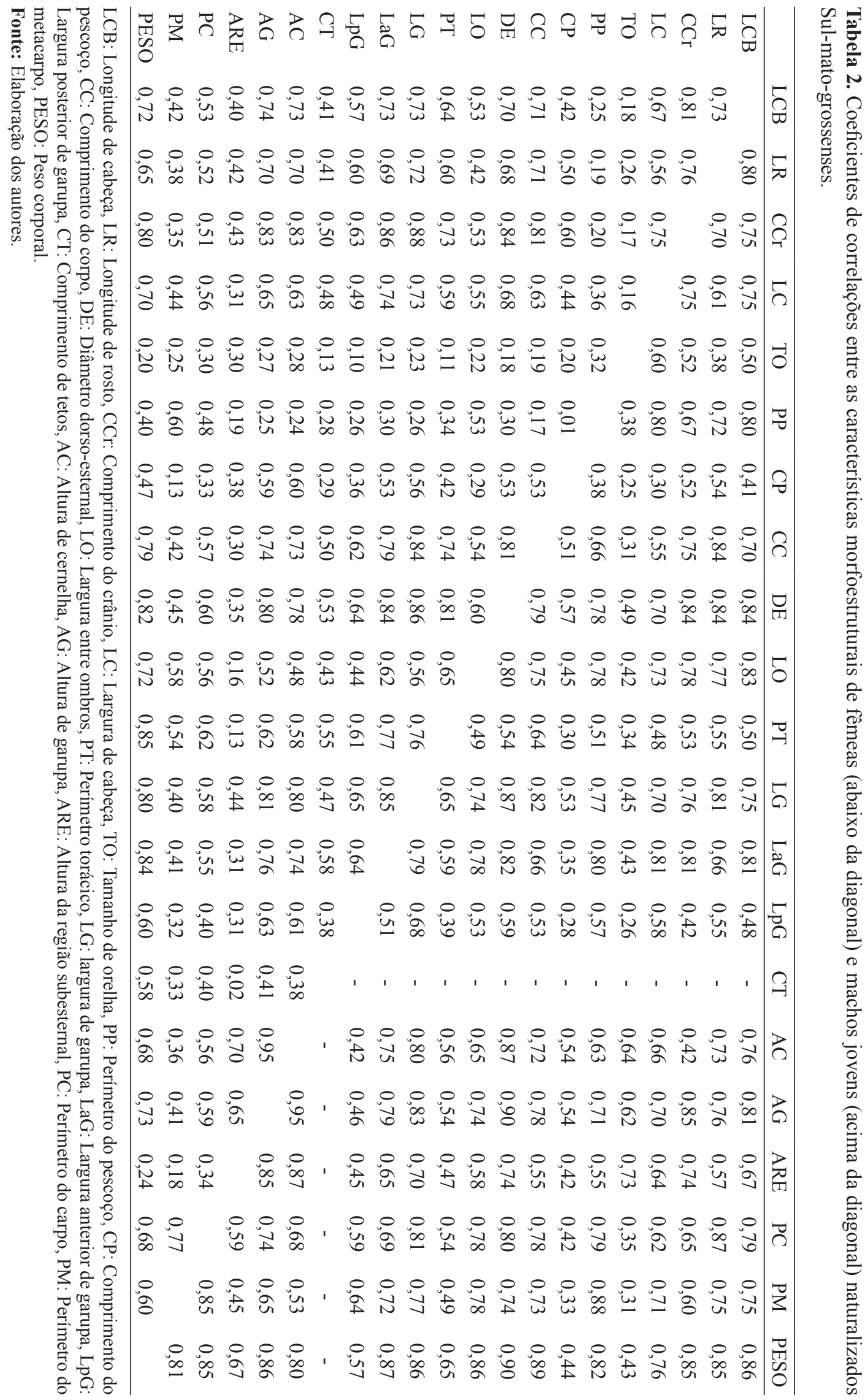


A região cefálica teve moderada a forte $(0,40$ - 0,88) associação com as variáveis mensuradas na região do tronco das fêmeas, exceto para $\mathrm{PP}$, que foi uma região pouco associada com as variáveis mensuradas na cabeça. A região cefálica é responsável pela expressão dos caracteres raciais (ABUD et al., 2011) e o pescoço pelos caracteres sexuais secundários (ROSA; ABREU, 2007), consequentemente, por serem responsáveis pela expressão de diferentes características foram pouco associadas. Porém, o CC, a LG e a $\mathrm{LaG}$ foram características mais fortemente associadas com a região cefálica.

As características da região cefálica apresentaram moderada a forte $(0,40-0,83)$ associação com as variáveis mensuradas nos membros das fêmeas. Diferentemente, o perímetro de metacarpo (PM) foi fracamente associado com a largura de rosto (LR) e comprimento do crânio $(\mathrm{CCr})$. A variável altura de cernelha $(\mathrm{AC})$ e altura de garupa (AG) são as características mais fortemente associadas à região cefálica das fêmeas de ovinos naturalizados Sulmato-grossenses. Em ovelhas Texel, criadas na parte alta do Pantanal há fraca associação entre LC e CCr com outras regiões do corpo (OLIVEIRA et al., 2007).

De modo geral, a região do tronco possuiu moderada associação com as variáveis mensuradas na região dos membros das fêmeas. Cabendo salientar que $\mathrm{AC}$ e AG possuem forte associação com $\mathrm{CC}, \mathrm{DE}, \mathrm{LG}$ e LaG em fêmeas do grupo genético de ovinos naturalizados Sul-mato-grossenses. Porém, CT e PP apresentaram pouca a nenhuma associação com a ARE.

A região do tronco, como o $\mathrm{PP}, \mathrm{CP}, \mathrm{LpG}$ e o $\mathrm{CT}$ mostraram moderada associação com peso corporal, diferentemente das outras variáveis que revelaram forte associação com peso corporal, fazendo desta região a que possuiu maior associação com o peso corporal em fêmeas do grupo genético naturalizados Sul-mato-grossenses. A AC, AG, CC e PT, características fortemente associadas com o peso corporal, são de interesse econômico, por estarem relacionadas com a produtividade animal (ABUD et al., 2011). Segundo Costa Júnior et al. (2006), a seleção de animais com pesos mais elevados à idade adulta pode resultar em aumento das medidas morfométricas e, consequentemente, do tamanho do animal.

Para a região cefálica com a região do tronco, nos machos jovens, observou-se que o comprimento do pescoço $(\mathrm{CP})$, a largura posterior de garupa (LpG) e o tamanho de orelha (TO) possuem fraca associação entre todas as variáveis mensuradas na região do tronco. Torres (2002) também observou resultados semelhantes para TO em ovelhas da raça Xisqueta. Porém, entre as outras características mensuradas observou-se moderada a forte associação entre essas regiões. Para a região cefálica com a região dos membros observou-se moderada a forte associação entre as características mensuradas.

Quando se relacionou a região do tronco com a região dos membros, em machos jovens, observouse que as características mensuradas apresentaram moderada a forte associação entre elas, exceto para PM com CP $(0,33)$. Porém, para o peso corporal, observou-se forte associação com todas as características mensuradas, exceto com tamanho de orelha (TO) na região cefálica; com comprimento do pescoço (CP) e largura posterior de garupa (LpG) na região do tronco e altura da região subesternal (ARE) na região dos membros em que apresentaram fraca associação.

Os valores de correlações foram positivos e fortes para peso corporal com a maioria das características morfométricas. De acordo com Costa Junior et al.(2006), por estes resultados pode-se supor a ocorrência de resposta correlacionada destas características com a escolha de uma característica de fácil mensuração como critério de seleção.

Os coeficientes de correlação de Pearson entre as características morfoestruturais foram positivos de moderado a fortepara $72 \%$ e $92,6 \%$ das características de fêmeas e machos jovens naturalizados Sul-mato- 
grossenses, respectivamente. Segundo Cuesta (2009), maiores coeficientes de correlação entre as características morfoestruturais implicam num rebanho mais harmônico para a morfoestrutura.

\section{Caracterização morfoestrutural de fêmeas de acordo com a classe de idade}

Nas fêmeas, observou-se maior coeficiente de variação para as características morfoestruturais
(Tabelas 1 e 3). Com o aumento da idade, nas classes II e III, observou-se que os coeficientes de variação apresentaram valores abaixo de $10 \%$, exceto para TO, LpG, CT, em todas as classes de idade (Tabela 3). Essa redução considerável na variação entre as fêmeas para as características morfoestruturais indicou que, após a estabilização do desenvolvimento, elas se tornaram mais homogêneas com relação à morfoestrutura.

Tabela 3. Média, erro padrão (EP) e coeficiente de variação (CV) de variáveis morfoestruturais, em centímetros, para fêmeas do grupo genético naturalizado Sul-mato-grossense de acordo com a classe de idade.

\begin{tabular}{lrrrrrr}
\hline \multirow{2}{*}{ Variáveis } & \multicolumn{2}{c}{$\begin{array}{c}\text { Classe I (n=110) } \\
\text { (dente de leite) }\end{array}$} & \multicolumn{2}{c}{$\begin{array}{c}\text { Classe II (n=93) } \\
\left(1^{\mathrm{a}} \mathrm{e} 2^{\mathrm{a}} \text { muda) }\right.\end{array}$} & \multicolumn{2}{c}{$\begin{array}{c}\text { Classe III (n=91) } \\
\text { (acima da 3 } 3^{\mathrm{a}} \text { muda) }\end{array}$} \\
\cline { 2 - 7 } & \multicolumn{1}{c}{ Média $\pm \mathrm{EP}$} & $\mathrm{CV}(\%)$ & \multicolumn{1}{c}{ Média $\pm \mathrm{EP}$} & $\mathrm{CV}(\%)$ & \multicolumn{1}{c}{ Média $\pm \mathrm{EP}$} & $\mathrm{CV}(\%)$ \\
\hline LCB & $30,80 \pm 0,26 \mathrm{c}$ & 8,90 & $32,80 \pm 0,16 \mathrm{~b}$ & 4,87 & $34,00 \pm 0,14 \mathrm{a}$ & 4,11 \\
LR & $12,75 \pm 0,15 \mathrm{c}$ & 12,92 & $13,91 \pm 0,09 \mathrm{~b}$ & 6,66 & $14,63 \pm 0,09 \mathrm{a}$ & 6,47 \\
CCr & $22,00 \pm 0,21 \mathrm{c}$ & 10,39 & $24,83 \pm 0,09 \mathrm{~b}$ & 3,75 & $26,14 \pm 0,09 \mathrm{a}$ & 3,56 \\
LC & $8,78 \pm 0,07 \mathrm{c}$ & 9,26 & $9,51 \pm 0,07 \mathrm{~b}$ & 6,89 & $10,07 \pm 0,06 \mathrm{a}$ & 5,76 \\
TO & $14,28 \pm 0,15 \mathrm{a}$ & 10,99 & $14,28 \pm 0,14 \mathrm{a}$ & 9,55 & $14,35 \pm 0,17 \mathrm{a}$ & 11,60 \\
PP & $28,21 \pm 0,34 \mathrm{a}$ & 12,93 & $28,25 \pm 0,34 \mathrm{a}$ & 11,87 & $29,05 \pm 0,28 \mathrm{a}$ & 9,30 \\
CP & $24,37 \pm 0,26 \mathrm{c}$ & 11,25 & $26,00 \pm 0,23 \mathrm{~b}$ & 8,85 & $27,17 \pm 0,25 \mathrm{a}$ & 8,87 \\
CC & $59,41 \pm 0,54 \mathrm{c}$ & 9,65 & $64,16 \pm 0,37 \mathrm{~b}$ & 5,68 & $68,07 \pm 0,41 \mathrm{a}$ & 5,92 \\
DE & $25,20 \pm 0,26 \mathrm{c}$ & 11,15 & $27,42 \pm 0,14 \mathrm{~b}$ & 5,16 & $29,70 \pm 0,17 \mathrm{a}$ & 5,73 \\
LO & $15,75 \pm 0,18 \mathrm{~b}$ & 12,42 & $16,25 \pm 0,14 \mathrm{~b}$ & 8,73 & $17,38 \pm 0,15 \mathrm{a}$ & 8,43 \\
PT & $71,15 \pm 0,79 \mathrm{c}$ & 11,73 & $75,80 \pm 0,38 \mathrm{~b}$ & 4,94 & $82,10 \pm 0,47 \mathrm{a}$ & 5,52 \\
LG & $18,92 \pm 0,20 \mathrm{c}$ & 11,22 & $21,00 \pm 0,11 \mathrm{~b}$ & 5,22 & $22,24 \pm 0,09 \mathrm{a}$ & 4,15 \\
LaG & $13,53 \pm 0,20 \mathrm{c}$ & 15,60 & $15,73 \pm 0,14 \mathrm{~b}$ & 8,75 & $17,76 \pm 0,12 \mathrm{a}$ & 6,80 \\
LpG & $4,85 \pm 0,09 \mathrm{c}$ & 19,71 & $5,60 \pm 0,06 \mathrm{~b}$ & 11,22 & $5,93 \pm 0,07 \mathrm{a}$ & 12,73 \\
CT & $1,52 \pm 0,04 \mathrm{c}$ & 27,38 & $1,70 \pm 0,03 \mathrm{~b}$ & 21,05 & $2,03 \pm 0,03 \mathrm{a}$ & 16,91 \\
AC & $60,24 \pm 0,52 \mathrm{c}$ & 9,14 & $64,83 \pm 0,26 \mathrm{~b}$ & 3,94 & $66,54 \pm 0,28 \mathrm{a}$ & 4,11 \\
AG & $61,87 \pm 0,55 \mathrm{c}$ & 9,42 & $66,37 \pm 0,29 \mathrm{~b}$ & 4,26 & $68,89 \pm 0,30 \mathrm{a}$ & 4,28 \\
ARE & $35,42 \pm 0,32 \mathrm{~b}$ & 9,45 & $37,50 \pm 0,28 \mathrm{a}$ & 7,40 & $36,55 \pm 0,23 \mathrm{a}$ & 6,25 \\
PC & $12,04 \pm 0,08 \mathrm{~b}$ & 7,13 & $12,09 \pm 0,06 \mathrm{~b}$ & 5,34 & $12,60 \pm 0,06 \mathrm{a}$ & 4,70 \\
PM & $7,22 \pm 0,68 \mathrm{~b}$ & 8,88 & $7,11 \pm 0,05 \mathrm{~b}$ & 6,86 & $7,44 \pm 0,04 \mathrm{a}$ & 5,60 \\
PESO & $27,25 \pm 0,68 \mathrm{c}$ & 26,25 & $31,51 \pm 0,49 \mathrm{~b}$ & 15,01 & $39,57 \pm 0,59 \mathrm{a}$ & 14,42 \\
\hline
\end{tabular}

LCB: Longitude de cabeça, LR: Longitude de rosto, CCr: Comprimento do crânio, LC: Largura de cabeça, TO: Tamanho de orelha, PP: Perímetro do pescoço, CP: Comprimento do pescoço, CC: Comprimento do corpo, DE: Diâmetro dorso-esternal, LO: Largura entre ombros, PT: Perímetro torácico, LG: largura de garupa, LaG: Largura anterior de garupa, LpG: Largura posterior de garupa, CT: Comprimento de tetos, AC: Altura de cernelha, AG: Altura de garupa, ARE: Altura da região subesternal, PC: Perímetro do carpo, PM: Perímetro do metacarpo, PESO: Peso corporal.

*Médias seguidas de letras diferentes, na linha, diferem entre si (Teste Tukey, $\mathrm{P}<0,05$ ).

Fonte: Elaboração dos autores. 
A idade foi o fator de maior fonte de variação entre as fêmeas. Observou-se que todas as características mensuradas aumentaram com a idade. Na região cefálica e a região do tronco observou-se crescimento proporcional e gradativo, exceto para tamanho de orelha (TO), perímetro do pescoço (PP) e largura entre ombros (LO) onde não há diferença significativa $(\mathrm{P}>0,05)$ com $\mathrm{o}$ aumento da idade, indicando que o comprimento da orelha tem um crescimento até a idade de um ano aproximadamente e o comprimento do pescozo e a largura entre ombros são regiões pouco influenciadas pela idade.

Para as características mensuradas na região dos membros, observou-se um crescimento gradativo para altura de cernelha (AC) e altura de garupa (AG). O peso corporal das ovelhas também apresentou crescimento gradativo com o aumento da idade, indicando que estas características têm desenvolvimento proporcional ao desenvolvimento do tronco. Observou-se maior desenvolvimento do carpo (PC) e metacarpo (PM) na classe III, do que nas classes I e II.

As características da cabeça das ovelhas apontam para um animal com cabeça relativamente maior que as raças ovinas Segureña (BLANCO et al., 1990) e Araneza (CASANOVA; VIDAL, 2007) e menor que da raça Xisqueta.

As médias para CC, LO, PT, LG, LaG, AC, AG e $\mathrm{PM}$ observadas são semelhantes às ovelhas da raça Segureña, caracterizada como raça para produção de carne e adaptada ao seu ambiente com tronco largo e profundo (BLANCO et al., 1990).

Todavia, as médias para CC, LO, AC, DE, PT, LG, PM foram inferiores das ovelhas da raça ovina Araneza (CASANOVA; VIDAL, 2007). De acordo com os mesmos autores, a raça Araneza não pode ser classificada como um animal com aptidão para produção de carne porque possui elevados índices leiteiros e motores. As ovelhas da raça Xisqueta (TORRES, 2002) apresentam médias superiores para PT, AC, AG, CC, DE e PM as encontradas neste trabalho.

Fêmeas da raça Texel, criadas no alto Pantanal (OLIVEIRA et al., 2007), apresentaram CC e LG igual das ovelhas naturalizadas do Pantanal. Porém, apresentaram médias superiores para LC, PT, PM, $\mathrm{LaG}$ e $\mathrm{LpG}$ e inferiores para $\mathrm{AC}, \mathrm{AG}, \mathrm{DE}$ e $\mathrm{CCr}$ as encontradas no presente trabalho.

As fêmeas naturalizadas Sul-mato-grossenses tiveram características morfométricas nas classes de idade, como CC, AG, AC e PT, com valores médios inferiores ao da raça Cabugi e superiores aos da raça Morada Nova pertencente ao semiárido do Estado de Alagoas (SILVA et al., 2007). Comparadas com ovelhas da raça Santa Inês, criadas nas microrregiões de Teresina e Campo Maior, no Estado do Piauí (COSTA JÚNIOR et al., 2006), observou-se que para AC, AG, CC e PT as ovelhas naturalizadas Sul-mato-grossenses foram menores na altura e tamanho corporal, no entanto, para LG as médias foram similares nas classes de idade.

Dimorfismo sexual para as características morfoestruturais

Os ovinos com aproximadamente um ano de idade não apresentaram dimorfismo sexual para as medidas cefálicas (Tabela 4). De acordo com Abud et al. (2011), as características raciais são expressas na região da cabeça dos animais. As medidas cefálicas dos ovinos naturalizados Sulmato-grossenses apresentaram baixa variação $(\mathrm{CV}$ : 8,76-13,02), indicando homogeneidade no grupo genético. 
Tabela 4. Média, erro padrão e coeficiente de variação (CV.) das características morfoestruturais, em centímetros, para animais jovens de ambos os gêneros em ovinos naturalizados Sul-mato-grossenses.

\begin{tabular}{lrrr}
\hline \multirow{2}{*}{ Variáveis } & \multicolumn{2}{c}{ Média \pm Erro Padrão } & CV(\%) \\
\cline { 2 - 3 } & \multicolumn{1}{c}{ Fêmeas $(\mathrm{n}=110)$} & Machos $(\mathrm{n}=42)$ & 9,46 \\
Longitude da cabeça & $30,80 \pm 0,26 \mathrm{a}$ & $31,23 \pm 0,52 \mathrm{a}$ & 9,92 \\
Comprimento do crânio & $22,00 \pm 0,21 \mathrm{a}$ & $21,70 \pm 0,28 \mathrm{a}$ & 13,02 \\
Longitude do rosto & $12,75 \pm 0,15 \mathrm{a}$ & $12,03 \pm 0,23 \mathrm{a}$ & 8,76 \\
Largura da cabeça & $8,78 \pm 0,07 \mathrm{a}$ & $8,87 \pm 0,10 \mathrm{a}$ & 10,5 \\
Tamanho da orelha & $14,28 \pm 0,15 \mathrm{a}$ & $14,11 \pm 0,19 \mathrm{a}$ & 13,50 \\
Perímetro do pescoço & $28,21 \pm 0,34 \mathrm{~b}$ & $30,11 \pm 0,64 \mathrm{a}$ & 10,35 \\
Comprimento do pescoço & $24,37 \pm 0,26 \mathrm{a}$ & $24,20 \pm 0,28 \mathrm{a}$ & 9,88 \\
Comprimento do corpo & $59,41 \pm 0,54 \mathrm{a}$ & $59,91 \pm 0,97 \mathrm{a}$ & 10,81 \\
Diâmetro dorso-esternal & $25,19 \pm 0,26 \mathrm{a}$ & $24,88 \pm 0,38 \mathrm{a}$ & 12,50 \\
Largura entre ombros & $15,75 \pm 0,18 \mathrm{a}$ & $16,24 \pm 0,31 \mathrm{a}$ & 11,92 \\
Perímetro torácico & $71,15 \pm 0,80 \mathrm{a}$ & $71,24 \pm 1,37 \mathrm{a}$ & 11,01 \\
Largura da garupa & $18,92 \pm 0,20 \mathrm{a}$ & $18,90 \pm 0,30 \mathrm{a}$ & 14,85 \\
Largura anterior da garupa & $13,53 \pm 0,20 \mathrm{a}$ & $13,22 \pm 0,25 \mathrm{a}$ & 27,95 \\
Largura posterior da garupa & $5,00 \pm 0,14 \mathrm{a}$ & $4,28 \pm 0,11 \mathrm{~b}$ & 8,83 \\
Altura da cernelha & $60,24 \pm 0,52 \mathrm{a}$ & $60,23 \pm 0,75 \mathrm{a}$ & 9,00 \\
Altura de garupa & $61,87 \pm 0,55 \mathrm{a}$ & $61,34 \pm 0,74 \mathrm{a}$ & 9,52 \\
Altura da região subesternal & $35,42 \pm 0,31 \mathrm{a}$ & $35,15 \pm 0,53 \mathrm{a}$ & 8,28 \\
Perímetro do carpo & $12,04 \pm 0,08 \mathrm{~b}$ & $12,66 \pm 0,19 \mathrm{a}$ & 9,96 \\
Perímetro do metacarpo & $7,22 \pm 0,06 \mathrm{~b}$ & $7,51 \pm 0,13 \mathrm{a}$ & 27,84 \\
Peso corporal & $27,25 \pm 0,68 \mathrm{a}$ & $29,56 \pm 1,40 \mathrm{a}$ &
\end{tabular}

Médias seguidas de letras diferentes, na linha, diferem entre si (Teste $\mathrm{F}, \mathrm{P}<0,05$ ).

Fonte: Elaboração dos autores.

Para as medidas no tronco observou-se que somente o perímetro do pescoço e a largura posterior de garupa apresentaram dimorfismo sexual nos animais. De acordo com Rosa e Abreu (2007), a observação de um pescoço mais grosso nos machos, é pertinente a expressão do hormônio da testosterona, que é responsável pela expressão dos caracteres sexuais secundários masculinos.

As diferenças observadas para largura posterior de garupa nas fêmeas teve relação com a adaptação delas para o nascimento do filhote. A LpG é uma característica ligada à reprodução, por isso a região pubiana foi mais desenvolvida nas fêmeas, diminuindo assim a probabilidade de partos distócicos (RODRÍGUEZ et al., 1990; ABUD et al., 2011). Vargas Junior et al. (2011b) não observaram diferenças entre cordeiros pantaneiros para comprimento corporal, perímetro torácico, largura de garupa e altura do posterior.

Para as medidas nos membros observou-se dimorfismo sexual somente nas regiões do carpo e metacarpo. Os machos apresentaram maior desenvolvimento nesta região pelo desenvolvimento ósseo superior ligado ao gênero (BLANCO et al., 1990; TORRES, 2002).

O peso corporal dos animais jovens não diferiu entre gênero, indicando que não houve dimorfismo sexual para esta característica nesta classe de idade (PAULINI et al., 2010; VARGAS JUNIOR et al., 2011b). Segundo Paulini et al. (2010), o dimorfismo foi mais acentuado em animais adultos, pela ação de hormônios sexuais, essa afirmativa está de acordo com os resultados apresentados por Blanco et al. (1990), Torres (2002) e por Costa Júnior et al. (2006). 


\section{Conclusões}

As ovelhas e machos jovens do grupo genético de ovinos naturalizados Sul-mato-grossenses apresentaram boa uniformidade morfoestrutural para a região cefálica e dos membros. A região do tronco apresenta maiores variações, indicando que esta região deve ser critério de seleção em programas de melhoramento.

Os coeficientes de correlação tanto em fêmeas quanto em machos jovens do grupo genético de ovinos naturalizados Sul-mato-grossenses apresentaram maior grau de conformidade para as características morfoestruturais formando um rebanho mais harmônico.

As ovelhas naturalizadas Sul-mato-grossenses apresentaram características morfométricas semelhantes a raças ovinas criadas no Alto Pantanal, no semiárido brasileiro e na Espanha. Caracterizaram-se por fêmeas com cabeça relativamente grande, com características do tronco proporcionais e bons aprumos.

As características da região cefálica e o peso corporal são semelhantes entre gênero em animais jovens. As características das outras regiões, como o perímetro do pescoço e largura posterior de garupa mensuradas na região do tronco, e para perímetro do carpo e metacarpo nos membros foram influenciadas pelo gênero nos animais jovens.

\section{Agradecimentos}

Ao Conselho Nacional de Desenvolvimento Cientifico e Tecnológico (CNPq) e ao Programa Nacional de Cooperação Acadêmica (PROCAD/ CAPES), pela concessão dos recursos para a realização da pesquisa.

\section{Referências}

ABUD, L. J.; GUIMARÃES, C. G.; PIMENTEL, C. M.; FIORAVANTI, M. C. S.; MARTINS, C. F.; SERENO, J. R. Morfometria corporal de novilhas nelore gestantes e não gestantes. In: CONGRESSO BRASILEIRO BUIATRIA, 9., 2011, Goiânia. Anais... Goiânia: Veterinária e Zootecnia, v. 18, p. 938-940, dez. 2011. Suplemento 3.

BLANCO, F.; GARCIA, V. D.; RUÍZ, F. A.; MIRA, M. da C. Estudio biométrico em la raza ovina Segureña. Archivos de Zootecnia, Córdoba, v. 39, n. 145, p. 249261, 1990.

CARNEIRO, H. A. Caracterização morfológica de ovinos no Brasil, Uruguai e Colômbia. Brasília, 2008. Dissertação (Mestrado em Ciências Animais) - Curso de Pós-Graduação em Ciências Animais. Universidade de Brasília, Brasília.

CASANOVA, P. M. P.; VIDAL, J. J. Análisis biométrico y funcional de la raza ovina aranesa. Pequeños Rumiantes, Espanha, v. 8, n. 2, p. 31-37, jul. 2007.

COSTAJÚNIOR, G. S.; CAMPELO, J. E. G.;AZEVÊDO, D. M. M. R.; MARTINS FILHO, R.; CAVALCANTE, R. R.; LOPES, J. B.; OLIVEIRA, M. E. Caracterização morfométrica de ovinos da raça Santa Inês criados nas microrregiões de Teresina e Campo Maior, Piauí. Revista Brasileira de Zootecnia, Minas Gerais, v. 35, n. 6, p. 2260-2267, 2006.

CUESTA, M. L. Uso de la caracterización para diferenciar y definir los RGAn. In: FEDERACIÓN ESPANHOLA DE ASOCIACIONES DE GANADO SELECTO FEAGAS/FAO. Bariloche: FAO, 2009.

GINÉS, R. Variación morfológica. In: ASTIZ, C. S. Valoración morfológica de los animales domésticos. Madri: Madri: Ministerio de Medio Ambiente y Medio Rural y Marino, 2009. cap. 5, p. 145-169.

GOMES, W. S.; ARAÚJO, Â. R.; CAETANO, A. R.; MARTINS, C. F.; VARGAS JUNIOR, F. M.; McMANUS, C.; PAIVA, S. R. Origem e Diversidade Genética da Ovelha Crioula do Pantanal, Brasil. In: SIMPOSIO DE RECURSOS GENÉTICOS PARA AMÉRICA LATINA Y EL CARIBE, 2007, Chapingo. Anais... México: Universidad Autónoma Chapingo, 2007. p. 322.

HERRERA, M.; RODERO, E.; GUTIERREZ, M. J.; PEFIA, F.; RODERO, J. M. Application of multifactorial discriminant analyses in the morphostructural differentiation of andalusian caprine breeds. Small Ruminant Research, Amsterdam, v. 22, n. 1, p. 39-47, 1996.

MARIANTE, A. da S.; ALBUQUERQUE, M. do S. M.; EGITO, A. A.; McMANUS, C. Advances in the Brazilian animal genetic resources conservation programme. Animal Genetic Resources Information, Cambridge, v. 25, n. 1, p. 107-121, 1999.

McMANUS, C.; FALCÃO, R. A.; SPRITZE, A.; COSTA, 
D.; LOUVANDINI, H.; DIAS, L. T.; TEIXEIRA, R. A.; REZENDE, M. J. M.; GARCIA, J. A. S. Caracterização morfológica de eqüinos da raça campeiro. Revista Brasileira de Zootecnia, Minas Gerais, v. 34, n. 5, p. 1553-1562, 2005.

OLIVEIRA, M. F. de; OJEDA FILHO, S. C. F.; HERNÁNDEZ, I.; LEITE, L. V.; SOUZA, J. C.; ABREU, U. G. P. de; SERENO, J. R. B. Avaliação fenotípica de ovelhas da Raça Texel criadas na parte Alta do Pantanal, Corumbá, n. 76, p. 24, 2007. Boletim de Pesquisa/ Embrapa Pantanal. Disponível em: <http://www.cpap. embrapa.br/publicacoes/online/BP76.pdf>. Acesso em: 03 jan. 2012.

PAULINI, F.; SILVA, M. C.; McMANUS, C.; FIORAVANTI, M. C. S.; VAZ, C. M.; SERENO, J. R. B. Estudo do dimorfismo sexual em cordeiros da raça crioula lanada. In: CONGRESSO BRASILEIRO DE RECURSOS GENÉTICOS, 1., 2010, Bahia. Anais... Brasília: Embrapa Recursos Genéticos e Biotecnologia, 2010. p. 19. CD-ROM.

RODRÍGUEZ, P. L.; TOVAR, J. J.; ROTA, A. M.; ROJAS, A.; MARTÍN, L. El exterior de la cabra Verata. Archivos de Zootecnia, Córdoba, v. 39, n. 1, p. 43-57, 1990.

ROSA, A. do N.; ABREU, U. G. P. de. Características que devem ser avaliadas para seleção ou compra de touros. Corumbá, 2007. 3 p. (Comunicado Técnico Embrapa Pantanal, n. 72). Disponível em: <http:// www.cpap.embrapa.br/publicacoes/download.php?arq pdf $=$ CT72 $>$. Acesso em: 03 jan. 2012.
STATISTICAL ANALYSIS SYSTEM INSTITUTE SAS. Statistical analyses system introductory guide for personal computers. Release. Cary NC: Sas Institute Inc., 2002.

SILVA, N. V.; FRAGA, A. B.; ARAÚJO FILHO, J. T.; CAVALCANTI NETO, C. C.; SILVA, F. L.; COSTA, P. P. S.; LIRA JÚNIOR, W. B. Caracterização morfométrica de ovinos deslanados Cabugi e Morada Nova. Revista Científica Produção de Animal, Piauí, v. 9, n. 1, p. 65-75, 2007.

TORRES, R. A. La Raza ovina Xisqueta: estudo biométrico y caracterización estructural de las explotaciones, Bellaterra. 2002. Tese (Doutorado em Produção Animal) - Curso de Pós-Graduação em Produção Animal, Universidad Autónoma de Barcelona, Barcelona.

VARGAS JUNIOR, F. M.; LONGO, M. L.; SENO, L. de O.; PINTO, G. dos S.; BARBOSA-FERREIRA, M.; OLIVEIRA, D. P. de. Potencial produtivo de um grupamento genético de ovinos nativos Sul-matogrossenses. PUBVET, Londrina, v. 5, n. 30, ed. 177, Art. 1197, 2011a. Disponível em: <http://www.pubvet.com. br/artigos_det.asp?artigo=1083>. Acesso em: $14 \mathrm{dez}$. 2011.

VARGAS JUNIOR, F. M.; MARTINS, C. F.; SOUZA, C. C.; PINTO, G. dos S.; PEREIRA, H. F.; CAMILO, F. R.; AZEVEDO JÚNIOR, N. P. Avaliação Biométrica de Cordeiros Pantaneiros. Revista Agrarian, Dourados, v. 4, n. 11, p. $60-65,2011$ b. 